\title{
The SSR System 7: A general encoding system with computerized transcription
}

\author{
GORDON R. STEPHENSON and THOMAS W. ROBERTS \\ The University of Wisconsin, Madison, Wisconsin 53706
}

\begin{abstract}
This report provides an overview of the wide variety of research problems to which the SSR System is being applied and outlines some of the changes in the system that these new challenges have brought about.
\end{abstract}

The SSR System is a general method for encoding data in real time for subsequent high-speed transcription by computer. The system includes a set of strategies for minimizing the task of the observer, a keyboard for encoding observations of ongoing events, an audio tape recorder for recording keyboard output, a high-speed playback tape deck, a signal conditioning circuit, a small computer, and software for transcription, timing, and organization of the encoded data. Since its publication (Stephenson, Smith, \& Roberts, 1975), the SSR System has been used to record the incidence, duration, coincidence, and sequence of behavior in laboratory and field studies of jellyfish, crayfish, crabs, spiders, fish, lizards, birds, jackals, monkeys, and humans. Zoologists, psychologists, sociologists, pediatricians, and psychiatrists have all found it applicable to their research problems. Users have usually taken advantage of the SSR System's open format and software definition of functions to tailor the structures of their keyboard entries to their particular research needs. The encoding process also allows the observer to record his subjects' vocalizations or his own supplementary voice notes on the parallel track of a stereo tape recorder.

Since our last report, the large data capacity and userdefinable features of the SSR System have been utilized to record an increasing variety of binary and digitized analog data from an expanding array of newly developed peripheral devices.

\section{NEW KEYBOARD DESIGN}

Keyboard entry of observed events is still the primary application of the SSR System. The SSR System 7 keyboard measures $9 \times 12 \times 2$ in. and weighs 4 lbs without

This work (Publication 17-017) of the Wisconsin Regional Primate Research Center) was supported by Grant RR-00167 from the National Institutes of Health. The authors thank the users for freely offering ideas that contributed to the continued development of the SSR System. The first author is Assistant Scientist, Wisconsin Regional Primate Research Center, 1223 Capitol Court, Madison, Wisconsin 53706. The second author is Lecturer, Department of Physical Education and Dance, Lathrop Hall, University of Wisconsin, Madison, Wisconsin 53706 . its detachable cover (Figure 1). The cover weighs $1 \mathrm{lb}$. Integrated circuits are used throughout. Three AA cells with an expected battery life of $1,000 \mathrm{~h}$ power the circuit. The time base is digitally derived from a $100-\mathrm{kHz}$ quartz crystal and is stable across a voltage range from 3 to $12 \mathrm{~V}$. The standard character set is $0-9$, $\mathrm{A}-\mathrm{Z}$, and $+,-, /, *, !,=, \$, @, \%$, ?, blank, and "segment," which can be either $<$ or $>$. The keyboard circuit is also used to encode data from the peripheral devices described below. All of the SSR System 7 devices described here have been developed by Semeiotic Systems Corporation of Madison, Wisconsin.

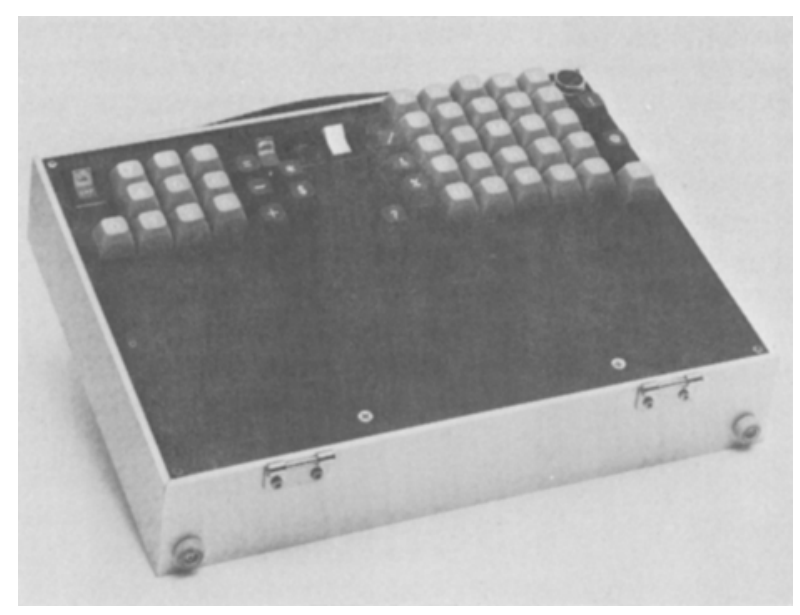

Figure 1. The SSR System 7 keyboard. Output jack is at upper right. Left to right, the rocker switches are POWER ON/ OFF, TAPE MOVE/STOP, SIGNAL SEND/STANDBY, and SEGMENT BEGIN/END. The TAPE switch utilizes the standard Uher microphone input circuit to control the PAUSE/OPERATE switch of the Uher tape recorder. The SIGNAL switch insures that data records begin and end with complete sweeps. The SEGMENT switch is a data key; depressing the upper half yields a $<$ to indicate BEGIN sample; depressing the lower half yields a $>$ to indicate END sample. The numeric subset is organized in a standard calculator format. The alphabetic subset is a 5 by 5 grid. By superimposing the grid on the study environment, it can be used grammatically to code location as well as to enter an action substring. The ? and $z$ keys are offset to enhance their use in error correction. See text. 


\section{THREE LOGICAL LEVELS OF ENCODING}

The 48 1-bit channels provided by the SSR System 7 method of encoding can be used at three different levels of logic. The three levels are software defined; no changes in the encoding or decoding hardware are necessary, but in some cases special peripheral devices are required.

\section{Encoding and Decoding}

Data collected with an SSR System 7 device are multiplexed 20 times/sec and phase encoded into a waveform (herein called a "sweep") that can be recorded on an audio tape recorder. Each of the 48 binary data channels is physically assigned to an ordinal position in the sweep. At the start of each sweep, the state (ON or OFF) of each of the channels is strobed into a 48-bit shift register. Each bit value is phase encoded as the contents of the register are shifted bit by bit and serially transmitted to the tape recorder.

Computer transcription of the SSR System 7 encoded record is divided into two stages. In the first stage, each sweep is decoded and compared to the last sweep that differed from its predecessors. If the current sweep does not differ, the sweep count is simply incremented and the program waits for the next sweep. If the current sweep does differ, it becomes the last sweep that differed and its contents and sweep count are saved for subsequent processing by the second stage of transcription. The sweep count is then incremented and the program waits for the next sweep. Thus, the sweep rate of $20 / \mathrm{sec}$ provides a time base that allows the data to be compressed on this first pass. The real time of every data entry is maintained to the nearest $.05 \mathrm{sec}$. Lastly, since the time base is encoded on the tape, it is independent of tape speed, and $1 \mathrm{~h}$ of data recorded at 1-7/8 ips (inches/sec) can be transcribed in 7-1/2 min by playing back into the computer at 15 ips.

Since the details of the second stage of transcription vary with the kind of data collected, description of this stage is presented with the descriptions of the data types.

\section{The Binary Level}

The keyboard can be used as a 48-channel event recorder, where each channel is assigned to a two-state switch that, in turn, stands for a unique category of event. The category is represented by the character on the key that operates the switch. The onset of an event in one of the categories is entered by pushing the appropriate key into its $\mathrm{ON}$ position. The termination is entered by pushing the key into its OFF position.

In the second stage of transcription of a binary-level record, each channel is assigned to a unique field in the output format. In the transcript, each line represents a sweep that differed from the last that was different. For any channel that changed from OFF to ON in the sweep, a + is printed in the corresponding field of the current line. Similarly, for any channel that changed from ON to OFF in the current sweep, $a$ - is printed. Channels that did not change in the sweep are represented by blanks. The sweep count is printed in a field at the extreme left. A corresponding array of data is also written onto a disk file for storage and further data processing.

The single-stroke entry of events at the binary level of logic is especially useful for very rapid coding of data, but the information load on the user is high with respect to the amount of time required to classify the observation and push the correct one of 48 keys. Hence, the temporal location of an uncommon code is likely to be late with respect to that of a more common one. Dr. Jeffery Baylis of the University of WisconsinMadison-Zoology Department is the only investigator currently using the keyboard in this manner. While doing so allows him to keep up with the interactions of fish, he is also limited to 48 codes for categorizing their behavior.

\section{The Digitized Analog Level}

At the second level of logic, channels can be grouped to serially encode digitized analog data that has been converted and formatted by the SSR System 7 ATD peripheral device. For example, the 48 bits can be divided into four 12-bit or six 8-bit channels. Again, at the first stage of transcription, only the sweeps that differed from their predecessors are saved (together with their sweep counts). At the second stage, the groups of bit values are processed as binary representations of the original analog values and are simply converted to their decimal equivalents for output in the user's format.

\section{The Grammatical Level}

At this level, the primary logical unit is the string of characters (the sequence of switches) rather than the single character or group of bit values. The structure of the character string is defined by a grammar or set of rules for keying in the codes that describe the observed event. At transcription, the salient aspect of a character's entry into the record is that the associated switch changed state: In contrast to the binary level, the direction of change is ignored. When a sweep is found to differ from the last that was different, the alphanumeric character assigned to the bit that changed is entered into the transcript. Since the order of characters is of critical importance in grammatical usage, change in more than 1 bit in a sweep must be regarded as a logical error. When multiple changes do occur in a single sweep, they are all enclosed within parentheses in the transcript.

To delimit the beginning of a string of characters that encodes an observed event, special characters, like,+- , $1, *$, and !, are software defined to function as carriagecontrol characters. Whenever the second-stage transcription program encounters one of these characters, a new 
line is begun in the transcript. All characters that are not defined as carriage-control characters are ordered into the line behind the most recently encountered line starter. Each of the line-starter characters can be further defined for other functions in data processing. For example, $a+$ is usually used to start entries that indicate the occurrence of a momentary event or the onset of a continuous event. A - is used to start entries that indicate the termination of a continuous event. Since the sweep count of the line starter is saved as the time frame of the entry, the strategy of having only a few characters to choose from at the beginning of an entry enhances the temporal accuracy of all entries with respect to one another regardless of the relative uncommonness of the characters that follow.

Even though entry of an observation may be slightly slower, most users have found it more advantageous to use the SSR System keyboard as a grammatical encoder than as a multichannel event recorder. In grammatical usage, the 10 switches assigned to the digits 0.9 can be used in 100 different two-character sequences to code 100 different two-digit names (00-99), and the 26 switches assigned to the letters A-Z can be used singly or in two-character sequences to stand for more than 600 different categories of behavior. Few investigators ever use more than a fraction of this capacity, but most take advantage of the full alphabet to mnemonically label their categories. Sequences of digits and letters and digits can then be strung together to code the subject and action and object of an event. Other characters, like $\$, @, \%$, =, and \& (or blank), can be used to delimit substrings within the line for coding proxemic relations of the subjects and objects and the locations of events in space.

\section{SOME APPLICATIONS}

\section{A Simple Grammar for the Water Test}

A very simple application of the SSR System keyboard as a grammatical encoder is described in our previous report (Stephenson, Smith, \& Roberts, 1975). The task is to record the onset and termination of drinking in a priority of access to water test of members of an enclosed troop of thesus monkeys after $22 \mathrm{~h}$ of water deprivation. The single source of water is constructed in a way that permits only one individual to drink at a time. Since the test is to determine the dependent rank order among the 50 individuals in the troop, the identity of the drinker also has to be recorded. We use a + to indicate the onset of drinking and two digits for the drinker's name. If drinking continues for less than $1 / 2 \mathrm{sec}$, the event is called a sip, which is defined as a momentary event of $1 / 2$-sec duration. If drinking continues more than $1 / 2$ sec, a $D$ is entered into the line to indicate that the entry is a drink, which is defined as a continuous event that requires specific termination. An error-checking subroutine in the data processing programs (after transcription) checks for a - as the next entry after a drink. If another + is entered before a drink is terminated, an error message is written into the processed data file. An error is also noted if a - follows a + that is not followed by a $D$, since a sip is defined as a momentary behavior and cannot be terminated but the $\mathrm{D}$ of a drink could have been forgotten. Since only one individual can drink at a time, its identification is entered in only the first sip or drink of each drinking bout. This convention reduces the number of key strokes and enhances the user's temporal accuracy in following the often rapid series of sips and drinks in a water test. Similarly, indicating the onset of both sips and drinks with $a+$, and only then distinguishing a drink from a sip by adding a $D$ to the line, enhances the accuracy of the event's temporal location in the test.

Using the SSR System for even this very simple situation has reduced the amount of work in preparing the results of the 1 -h test for quantitative data processing from about $10 \mathrm{~h}$ to about $1 \mathrm{~h} 35 \mathrm{~min}$, including complete verification of the printout against the parallel voice notes and editing any corrections into the manuscript. Thus, we have been able to afford to follow the dynamics of change in dependent rank relations with a water test once each week for the last $3^{1} / 2$ years, while continuing to concentrate on the details of social interaction in our daily observations. A series of reports is in preparation.

\section{A Complex Grammar for the Pair Test}

The basic notions of grammatical processing have recently been applied to the problem of coding dyadic encounters (Stephenson, Note 1). The structural details of the problem became evident in the course of focal animal studies of monkeys in troops at the Vilas Park Breeding Research Facility of the Wisconsin Regional Primate Research Center and in Lamb's (in press) studies of human infants in the presence of their mothers and fathers, both singly and together, at the Waisman Center on Mental Retardation and Human Development, University of Wisconsin. A software solution in the form of a general dyad processor, Program PARSYN (for pair syntax), was finally written for data collection in a collaborative study with Goldfoot and Essock-Vitale (Note 2). Program PARSYN reads the definitions of the user's code from one file and then operates on the user's raw SSR System transcript (in a second file) to check, complete, reformat, and write the processed entries into a third file for subsequent data processing by other programs. PARSYN operates on the data by following the values assigned to the properties of the category in the current line of data.

For example, $A$ is the code for approach in the set of codes for recording observations of rhesus or stumptail macaques in the sex pair tests in Goldfoot's studies (see Goldfoot, Essock-Vitale, Asa, Thornton, \& Leshner, in press). The first coding property (CP1) for the 
category of approach is A, its mnemonic alphabetic label. CP2 indicates whether the subject of an entry with this code is required or can be assumed. For A the subject can be assumed. Since either the male (1) can approach the female (2) or the female can approach the male, the subject is not restricted (CP3). On the other hand, the subject of an entry indicating an ejaculation is restricted in this test. If the subject is not required, the value of CP4 is the name of the assumed or default subject. Since males usually approach females in these tests, rather than vice versa, the value of Code A's CP4 is one. The object of an approach in the pair test is not required (CP5), and the assumed object (CP6) is defined as the opposite of the subject.

Definition of the coding properties reduces the number of descriptive elements that must be entered when an approach is observed. Since approach is a commonly occurring category, it is labeled, in accordance with Zipf's law, with a single letter rather than two. The user can now enter his observation of a male approaching a female by simply keying in $+A$ rather than $+1 A 2$. Program PARSYN interprets the $+A$ as $+1 A 2$ and writes the completed and uniformly formatted entry into a disk file for later processing. If the female had approached the male, $+2 \mathrm{~A}$ would have been enough to yield $+2 \mathrm{~A} 1$ in the processed data. The entries $+1 \mathrm{~A}$, $+1 \mathrm{~A} 2$, and $+2 \mathrm{~A} 1$ would have also been accepted by PARSYN, but the entry +A1 would have resulted in an error message (on the processed file) because the assumed subject was defined as 1 and the assumed object was defined as the opposite of the subject. As demonstrated here, the value of CP6 controls both the completion of the object (if so defined) and its logical appropriateness.

Other coding properties control PARSYN's management of entries that indicate the onset or termination of continuous codes. These properties permit termination of an ongoing category by the entry of a mutually exclusive category of behavior in a line that starts with a + (indicating the occurrence or onset of the mutually exclusive event) as well as by specific termination through entry of the original code in a line that starts with a - . When PARSYN detects a mutual exclusion, the termination of the mutually excluded category is inserted into the processed data file. These kinds of properties also allow extensive error checking, for example, for termination of a code that is not on (or is not defined as continuous) or for multiple onsets of a code without intervening terminations. For some codes, such as G for grooming, two instances of the category can be ongoing simultaneously (i.e., the male can be grooming the female while the female is grooming the male). In this case, the values of the coding properties lead PARSYN to check whether the subjects of the two onsets are different. If they are different, an error message is written into the processed file.

The capacity to define coding properties that indi- cate whether a category is continuous and whether it is mutually exclusive with other categories (and under what conditions) removes a large burden from the observer while he is entering his observations on the keyboard. It allows the observer to simply record what he sees as he sees it, without requiring that he remember a lot about what he has already seen and entered. For example, if the male grooming the female grooming him suddenly leaves, the observer only has to enter $+1 \mathrm{~L}$, rather than $+11,-1 \mathrm{G}$, and $-2 \mathrm{G}$. The $+1 \mathrm{~L}$ is all that is needed because $L$ is defined as a mutual terminator of $G$ in a way that overrides any requirement for agreement of the subjects" identities in the terminator and terminated entries. In the pair test situation, one subject leaving another is mutually exclusive with either one of the individuals grooming the other (self-grooming is a different category in the code and is labeled SG). This removal of some of the burden markedly enhances the observer's accuracy in recording social interactions in the pair test.

For errors that do occur and are sensed in the course of being entered, several conventions for immediate correction are designed into the observer's grammar and Program PARSYN. For example, a ? is a within-line grammar character that is used to delete all characters back to, but not including, the last line starter. This convention preserves the line starter so that the corrected codes following the ? will be associated with the original time frame of the entry. In the case where the next character after a ? is a line starter instead of a within-line character, PARSYN deletes the previous line starter and all of the characters between it and the line starter immediately after the ?. Another kind of immediate correction is called replacement. In PARSYN, \% is used to start a within-line substring of characters that modifies the action. A mistaken modifier can be corrected by simply entering the \% again followed by the corrected code before a new line is started. A third convention allows the user to correct the current syntactic unit (subject, action, or object) that he has either partially entered or just completed. If, for example, he has entered the first letter of a two-letter action code and it is a mistake, the user can simply enter the $Z$ to "zero" the current action code and continue the string of characters with the correct code. Here, this convention would have saved reentering the subject of the event, which the ? would have deleted, given that it was not assumed. These conventions seem to fit comfortably into the language-like stream of code that PARSYN facilitates, and they save time both during and after the observation session, especially by reducing the amount of editing that might otherwise have to be done prior to quantitative analysis of the data. Lastly, since PARSYN corrects the errors, checks all of the raw data against the user's code properties and completes those entries with assumed values, and reformats the syntactic elements of the entry into a uniform set of 
data fields, data processing programs can be relatively simple and primarily concerned with quantitative analysis rather than missing or erroneous data.

\section{Other Grammars}

The general principles of PARSYN are being further developed to handle the general case of recording social interactions among members of large social groups. The fact that the character set and all character functions are software defined and that the format for entering data is completely open leaves few limitations to hamper the user's imagination. For example, in some grammars $=$ is a within-line delimiter preceding the orientation indices of the subjects and objects of the statement, but in PARSYN it is a line starter that stands for the same entry as last entered and then repeated at the new time frame. In the new general grammar, this function is being expanded to facilitate the entry of repeated couplets, as might occur in play sequences between mother and baby. Thus, $=2$ after two lines of code for mother pat baby, baby laugh results in the processor inserting the completed and formatted form of the previous couplet, mother pat baby, baby laugh. The general grammar also borrows PARSYN's convention of ${ }^{*}$ as the line starter of an entry that began at the same time as the previous line. Lastly, the general grammar allows entry of multiple subjects, multiple actions, and multiple objects in a single line, which makes the whole process of recording ongoing social behavior an even more language-like experience in which "the fingers do the talking."

Even at the simpler level of definition of the character set, the flexibility of the SSR System has facilitated grammatical entry of observations. For example, Dr. Betsy Lozoff of the Department of Geographic Medicine at Case Western Reserve University School of Medicine found, after she was in the field among members of a preagricultural society in southern India at a location about a $1 / 2$-day run from the nearest electrical outlet, that two of her single-letter codes were used much more frequently than expected. To facilitate their rapid entry, she simply pried the key caps off their switches and moved them to a position below the place where her right index finger was normally poised between key strokes. She moved the displaced key caps to where the other two had been. Upon return from the field, Lozoff simply changed the data statement that assigns the characters to sweep positions in the second stage of transcription. The grammar processor still finds the correct character in the correct place in Lozoff's data, but the data have been enhanced by the fact that she can make the entries faster with those particular characters in their new locations on the keyboard.

\section{Combining Binary and Grammatical Data}

Several users have suggested that the capacity to record the ON/OFF status of remotely monitored binary channels in parallel with their grammatically structured entries of ongoing behavior would be very useful. For example, photocell circuits can be used to monitor the passage of individuals at specific locations in the study environment. Tripping the photocell circuit can be coded in two ways. If the movement through the light beam is expected to be slow, the state of the circuit (made or broken) can be encoded directly as $\mathrm{ON}$ and $\mathrm{OFF}$. And, in this case, the duration of passage can be recorded to the nearest $.05 \mathrm{sec}$. If passage is expected to be of very short duration (less than $.1 \mathrm{sec}$ ), interruption of the light beam can be used to drive a bistable (flip-flop) circuit with a time window appropriate to the typical duration of passage. In this case, that state of the flip-flop would be encoded and passages could only be counted. Both of these methods would yield interpassage intervals.

The capacity to record binary data in parallel with grammatically structured strings has been designed into the SSR System 7B keyboard. This model keyboard comes with five input jacks. Each jack is connected to one of the five sweep positions that is normally assigned to one of the alphabetic characters $U$ through $Y$. Insertion of the plug from an external binary channel into the jack usurps the corresponding character's sweep position and turns it over to the binary channel. Semeiotic Systems Corporation's binary input device (BID) provides five bistable channels for this kind of data. Each channel of the BID has adjustable voltage windows that enable the user to accommodate the channel to the output signal of his particular monitoring device. For transcription, the user simply declares the usurped character as a special line starter that does not interrupt the assembly of the current line but that is written in the transcript at the appropriate temporal location.

In collaboration with Dr. L. M. Passano and Mr. Kenneth G. Olesen of the University of Wisconsin Department of Zoology, we have recently applied the capacity for combining binary and grammatical data to Passano's polygraph records of electrophysiological activity in jellyfish. Before this application, the records were read with a manually operated viewer that counted the travel of the polygraph paper past a registration point. When the tracing of one of the four channels indicated activity, the waveform was classified, the onset of the waveform was manually aligned with the registration point, and the counter value was recorded. A large part of this tedious and time-consuming task has now been automated by using the activation of a polygraph pen to drive one of the BID channels, which, in turn, changes the state of the corresponding sweep position. Thus, all of the timing information from the four channels can be recorded at the usurped character positions on the keyboard, while environmental changes and the ongoing behavior of the jellyfish can be grammatically coded with the remaining keys. 


\section{Analog Data}

Rather than record the presence or absence of a signal, some users want to record the value of its voltage, where the value corresponds to some continuous measure of light level, pressure, temperature, or physiological parameter. To accommodate these users, an analog-to-digital peripheral device that converts signal amplitude $(0-5 \mathrm{~V})$ into its 12-bit binary equivalent has been developed. The Semeiotic Systems Corporation ATD peripheral converts and formats values in four parallel 12-bit channels for encoding by an SSR System 7A keyboard 10 times/sec.

The Model 7A keyboard has two modes of operation. In regular mode it operates as described above (see section on new keyboard design) to encode the states of the key switches 20 times/sec. In alternate mode it encodes the states of the key switches every other 20th of a second and encodes the digitized values in the ATD registers each intervening 20th of a second. The two kinds of sweeps are distinguished by the value of the 49th bit, called the signature bit. This sweep position is not available to the keyboard operator, but it is available for hardware and software options. In the Model 7 keyboard, the 49th bit is hardwired as an ON or available for the interval timer option described below. In the Model 7A, the value alternates as ON for the keyboard data and OFF for the digitized analog data. The alternate mode of operation yields an effective sampling rate of $10 / \mathrm{sec}$ for each type of data. Many users of the Model 6 keyboard (described in detail in Stephenson et al., 1975), which had an effective sampling rate of $10 / \mathrm{sec}$ due to some hardware and software limitations on grammatical usage that have been corrected in the Model 7 , found that this rate was very adequate to record their keyboard data without logical errors in the grammatical structures of their character strings.

The first application of an SSR System 7 keyboard with these capabilities is to follow the flight path of red-tailed hawks while they forage over open country. Each of the two observers working with Mr. Michael DeJong in the UW-Madison Zoology Department visually follows the hawk with a spotting scope that is equipped with cross hairs. Each scope has two highprecision potentiometers, one to indicate azimuth and one to indicate altitude of the scope's line of sight. The scopes are a base distance apart (as determined by terrain and desired degree of accuracy) and are calibrated at the beginning of each session by being sighted on several points in the environment that can be seen from both scope locations. It is best if the scopes can also be sighted on each other. A rocker switch on each scope controls the state of a binary channel that usurps one of the U.Y characters on the keyboard and is used to indicate whether the observer is "on the bird" or "off the bird" with his scope. The third observer enters his observations of environmental conditions, behavior, and habitat under the flight path on an SSR System 7C keyboard. This model of the keyboard combines the analog and tinary capabilities into one package. Lastly, throughout the recording of this complex data set, the parallel track of the stereo tape recorder is always available for voice comments on protocol and unexpected events.

At the first stage of transcription, the value of the 49th bit of each sweep is used to control the flow of program execution so that the current sweep is compared to the last of its kind that was different from its predecessors. If the current sweep differs, the sweep count and contents are saved for subsequent processing by the second stage of transcription. The two kinds of sweeps are kept distinct throughout data processing by using the value of the 49 th bit as the value of the sign bit of the sweep count saved.

After transcription, the calibration values and the digitized analog data are used to compute the flight path of the hawk by triangulation when the values of the binary channels indicate that both observers are on the bird with their scopes. Whenever only one observer is on the bird, the flight path is interpolated. When both are off the bird, the digitized analog data are ignored. Where the flight data are adequate, the path can be examined with respect to the behavioral and ecological data that were entered grammatically on the keyboard by the third observer.

One further note about the 49th bit: An interval timer option that generates an audible beep tone through either a small speaker or a headphone is available for SSR System 7 keyboards. The beep cues the observer to enter a sample of some particular kind of data once each interval. The interval length is selectable. In one application, the beeper reminds the observer to record the location and orientation of all adult males in the troop once each $10 \mathrm{~min}$ in the observation period. In the Model 7 and Model 7B keyboards, this option switches the state of the 49th bit. In the Model 7A and Model 7C keyboards, where the value of the 49th bit is the signature of the data type, the occurrence of the beep tone can be entered through one of the BID channels. The change in state of the beeper bit can later be used to check whether the sample was actually taken.

\section{Broad Spectrum Analog Data}

A final example of the adaptability of the SSR System 7 to real-time data collection problems in laboratory and field research was conceived recently in the course of conversation with Dr. Emil Menzel of the State University of New York at Stony Brook. While this application is still in the design stage, an outline of our approach may prove useful to others. Menzel's marmosets range freely within a large section of a greenhouse filled with plants and perches. In this environment the marmosets vocalize a lot. A detailed sound spectrogram analysis of their vocalizations has been 
completed. The problem now is to record long continuous samples of their vocalizations in a way that the sounds can be examined by sequence, lag, and time series techniques for dependency and pattern that might provide insight into their social function.

Our collaboration with Menzel has suggested the following approach. Microphone radio collars used in studies of bat vocalizations would be placed on the marmosets. The signals from each collar would be received by a separate radio channel. The signal in each channel would be presented to a set of bandpass filters. The frequency windows of the nonoverlapping bands would be adjusted to partition the frequency spectrum of marmoset vocalizations into eight parts that optimally discriminate the various signal forms their vocalizations manifest. A new SSR System 7 peripheral device, the broad spectrum digitizer (BSD), now being developed by Semeiotic Systems Corporation, would sample six channels of eight bands 10 times/sec. Where sound energy was present in the band during the sampling interval, the corresponding bit of a 48-bit register would be set. In the same way in which the digitized analog channels and the state of the keys were alternately encoded in the Model 7A and Model 7C key. boards, the Model 7D keyboard would alternately encode the digitized spectral data and the states of the keys. The keys would be used to code observations of social behavior, proxemics, and location of the individual marmosets as contextual background for the analysis. Taking advantage of the enormous data capacity of magnetic tape, the encoding signal would be recorded on 2,400 - or 3,600-ft lengths that provide up to $6 \mathrm{~h}$ of continuous data per side.

At transcription, the two kinds of data would be kept separate until a modified version of Program COMBO (see Stephenson et al., 1975) could print out the coded observations in temporal registration with the digitally reconstructed signal forms. The digital representations can also be evaluated by analysis programs for frequency, duration, and temporal patterning of particular signal types within and across the individual marmosets. Lastly, a comparison of the printout with the vocalizations recorded on the parallel stereo track would further refine the analysis. We believe that a similar approach would also be useful in the analysis of the temporal structures of utterances in studies of human communication.

\section{IMPLEMENTATIONS}

The SSR System was originally implemented by Dr. Daniel P. B. Smith on a Linc II computer in the Laboratory Computer Facility at the University of Wisconsin in 1968. The computer's size and speed limited playback to four times the rate at which the data were recorded on tape.

In 1972 Smith implemented a completely new ver- sion of the SSR System on a new Datacraft 6024/5 in the BOZO (Botany-Zoology) Computer Facility at UW-Madison. At that time, BOZO had 16K 24-bit words of core memory running as a dedicated machine under Datacraft's disk operating system. This configuration was fast enough to permit playback at eight times the recorded speed. While this rate required a special high-speed tape deck, playback time was reduced from $15 \mathrm{~min}$ to $7 \frac{1}{2} \mathrm{~min}$ per hour of real-time data, a savings that more than justified the investment, given the number of SSR System users in the UW Department of Zoology.

These first two implementations used a signal conditioning circuit and an analog-to-digital channel to get the data that had been encoded on the field data tape into the computer. The automatic gain control, high-pass filter, and Schmitt trigger of the conditioning circuit served to compensate for distortions of the encoding signal that normally occur in the course of recording and playback. The output of the Schmitt trigger was then digitized by the analog-to-digital converter for evaluation by the decoding subprogram in the first stage of transcription. After the logic of the subprogram was synchronized with the beginning of the sweep, the state of each data position in the sweep was decoded and stored in sequence until the current sweep was completely decoded and ready for comparison with the last sweep that was different from its predecessors.

While the decoding process remains the same, major simplifications in presenting the playback signal to the computer have been made. The conditioning circuit has been reduced to a window comparator. The analog-to-digital converter has been eliminated by presenting the TTL-compatible output of the comparator to the computer as the value of the sign bit of a memory location. Where this cannot be done directly, some additional circuitry is used to format the data for input through a digital or parallel port.

Three new implementations have used this approach. At the Waisman Center on Mental Retardation and Human Development, the Datacraft 6024/5 operates in timesharing mode under Datacraft's disk management system. This mode precludes the approach to transcription that had been implemented at $\mathrm{BOZO}$, which required a dedicated machine. Mr. Bruce Anderson of the Waisman Center utilizes a microprocessor to decode and compare sweeps. When a sweep differs from the last that was different, its contents and sweep count are sent to the Datacraft on a high-level priority interrupt basis and stored on a file for subsequent processing by the second-stage transcription program.

The simpler approach has also been used by Mr. Robert Philbim to implement the SSR System 7 on a PDP-11/20 at the Wisconsin Regional Primate Research Center. The output of the comparator is sent directly to the sign bit position of a DR-11 general inter- 
face board. This standard plug-in board has been assigned to a memory location that can be addressed and evaluated by the decoding program. Sweeps that differ are stored with their sweep counts on DECtape or disk for subsequent processing.

The simpler solution has been used by Mr. Douglas Boyle and Mr. Mark Morris in the Electrical Engineering and Biology Departments at Stanford University to develop a general SSR System decoder. The decoder is designed to take advantage of remote terminals and low-overhead timesharing systems generally available on larger university-wide computers. Records can be transcribed at real time or four times recorded speed, depending on whether the user is transmitting the sweeps that differ over a modem at 300 baud or directly into a computer at 9,600 baud. The decoder is also commerically available.

\section{AVAILABILITY}

SSR System 7 devices are available from Semeiotic Systems Corporation, 13 Homestead Court, Madison, Wisconsin 53711. The basic Model 7 keyboard is currently (August 1977) priced at $\$ 650$. Other prices are available upon request. Listings of system sof tware are available at no cost in the hope that other users will feel free to share their applications software with each other. Arrangements for special applications consulting can be made. Semeiotic Systems Corporation and the authors welcome any criticisms or suggestions that will allow the SSR System to better serve the needs of the research community.

\section{REFERENCE NOTES}

1. Stephenson. G. R. Concepts for coding dyadic encounters. Piper prestnted at the meetings of the American Society of Primatchogists. A pril 1977. Seattle. Washington.

2. Stephenson, G. R., Goldtoot, D. A.. \& Essock-Vitale. S. M. PARSYN: A computer-compatible grammar for coding diadic ancominers. In preparation.

\section{REFERENCES}

Goldfoot, D. A., Essock-Vitale, S. M., Asa. C. S.. Thornton. J. E.. \& Leshner. A. E. Anosmic male rhesus monkeys continue to display cyclic copulatory behavior. Solence, in press.

LAMB. M. E. Infant social recognition and "second order" effects. Infant Behavior and Development, in press.

Stephenson, G. R., Smith, D. P. B., \& Roberts, T. W. The SSR System: An open format event recording system with computerized transcription. Behavior Research Methods \& Instrumentation, 1975, 8, 259-277.

(Received for publication August 9.1977; accepted August 22.1977.) 